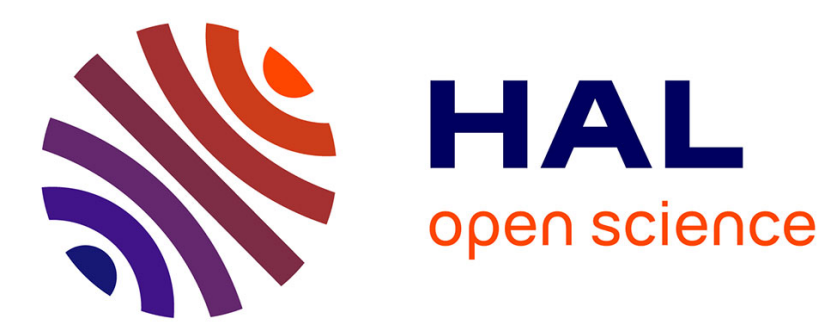

\title{
Haptic guides in cooperative virtual environments: Design and human performance evaluation
}

Sehat Ullah, Paul Richard, Samir Otmane, Mickael Naud, Malik Mallem

\section{To cite this version:}

Sehat Ullah, Paul Richard, Samir Otmane, Mickael Naud, Malik Mallem. Haptic guides in cooperative virtual environments: Design and human performance evaluation. 2010 IEEE Haptics Symposium, Mar 2010, Waltham, MA, United States. pp.457-462, 10.1109/HAPTIC.2010.5444616 . hal00649022

\section{HAL Id: hal-00649022 \\ https://hal.science/hal-00649022}

Submitted on 13 Nov 2013

HAL is a multi-disciplinary open access archive for the deposit and dissemination of scientific research documents, whether they are published or not. The documents may come from teaching and research institutions in France or abroad, or from public or private research centers.
L'archive ouverte pluridisciplinaire HAL, est destinée au dépôt et à la diffusion de documents scientifiques de niveau recherche, publiés ou non, émanant des établissements d'enseignement et de recherche français ou étrangers, des laboratoires publics ou privés. 


\section{Haptic Guides in Cooperative Virtual Environments: Design and Human Performance Evaluation}

\author{
Sehat Ullah* \\ IBISC Laboratory, \\ University of Evry France
}

\author{
Paul Richard ${ }^{\dagger}$ \\ LISA Laboratory, \\ University of Angers France
}

\author{
Samir Otmane \\ IBISC Laboratory, \\ University of Evry France
}

Malik Mallem II

IBISC Laboratory,

University of Evry France

\author{
Mickael Naud ${ }^{\S}$ \\ LISA Laboratory, \\ University of Angers France
}

\begin{abstract}
In this paper we simulate the use of two string based parallel robots in cooperative teleoperation task. Two users standing in front of a large screen operate each robot. We propose two haptic guide models, and investigate their effects on cooperation, co-presence and users performance. In addition we also examine the effect of simple force feedback in cooperative work. Ten volunteer subjects had to cooperatively perform a peg-in-hole task. Results revealed that haptic guides have a significant effect on task execution. They not only increase users performance but also enhance the sense of copresence and awareness. Our investigations will help in the development of teleoperation systems for cooperative assembly, surgical training and rehabilitation systems.
\end{abstract}

Index Terms: Computer Graphics [1.3.6]: Methodology and Techniques-Interaction Techniques

\section{INTRODUCTION}

The successful advancements in the field of high quality computer graphics and the capability of inexpensive personal computers to render high-end 3D graphics in a more realistic manner has made virtual reality feasible to be used in many areas such as industrial design, data visualization, medical training $[21,18,22,8]$, textile and fashion [13], assembling and education [2].

Human beings often perform their work (from simple to complex) in a collaborative manner, that is why VR scientists initiated the development of virtual environments (VEs) supporting collaborative work. A CVE is a computer generated world that enables people in local/remote locations to interact with synthetic objects and representations of other participants within it. The applications of such environments are in military training, telepresence, collaborative design and engineering, entertainment and education. Interaction in CVE may take one of the following form [15]:

- Asynchronous : It is the sequential manipulation of distinct or same attributes of an object, for example a person changes an object position, then another person paints it. Another example is, if a person moves an object to a place, then another person moves it further.

- Synchronous : It is the concurrent manipulation of distinct or the same attributes of an object, for example a person is

\footnotetext{
*e-mail: sehat.ullah@ibisc.univ-evry.fr

†e-mail:paul.richard@univ-angers.fr

†e-mail:samir.otmane@ibisc.univ-evry.fr

§e-mail:mickael.naud@univ-angers.fr

Ile-mail:malik.mallem@ibisc.univ-evry.fr
}

holding an object while another person is painting it, or when two or many people lift or displace a heavy object together.

The concurrent manipulation is also termed as Cooperative Manipulation or Cooperative work.

In order to carry out a cooperative task efficiently, the participants need to feel the presence of others. Communication among the participants during the cooperative task is also essential. The communication may be verbal or non verbal such as pointing to, looking at or even through gestures or facial expressions. Similarly the participants must have a common protocol for task execution. The design and implementation of a system with these capabilities have really been a challenging job for the researchers.

We simulate the use of two string based parallel robots in cooperative teleoperation task. Two users standing in front of a large screen operate each robot. We propose two haptic guide models, and investigate their effects on cooperation, co-presence and users performance. Our investigations will help in the development of teleoperation systems for cooperative assembly, surgical training and rehabilitation systems.

This section is followed by the related work. Section 3 describes the proposed "attractive and speed control haptic guides. Section 4 presents user experimentations and results analysis. Section 5 gives conclusion and some tracks for future work.

\section{Related Work}

A lot of work has already been done in the field of CVE, for example MASSIVE provides a collaborative environment for teleconferencing [9]. Most of this collaborative work is pertinent to the general software sketch, the underlying network architecture [25] and framework [1].

Basdogan et al. have investigated the role of force feedback in cooperative task. They connected two monitors and haptic devices to a single machine [3]. Similarly, sallnas et al. have reported the effect of force feedback over presence, awareness and task performance in a CVE. They also connected two monitors and haptic devices to a single host [23]. A heterogeneous scalable architecture that supports haptic interactions in collaborative tasks has been proposed [24]. But all these systems use force feedback only for realism and not for guidance in collaboration. Other important works that support the cooperative manipulation of objects in a VE include $[11,12]$ but all these systems require heavy data exchange between two nodes to keep them consistent. Haptic guides have successfully been used for 3D object selection in large scale VEs [27]. McSig is a multimodal teaching and learning environment for visually-impaired students to learn character shapes, handwriting and signatures collaboratively with their teachers. It combines haptic and audio output to realize the teacher's pen input in parallel non-visual modalities [17]. CHASE (Collaborative Haptics And Structured Editing),is a synchronous structured drawing tool. It provides telepointers and allows users to simultaneously work on a large canvas while each maintaining a separate view of 


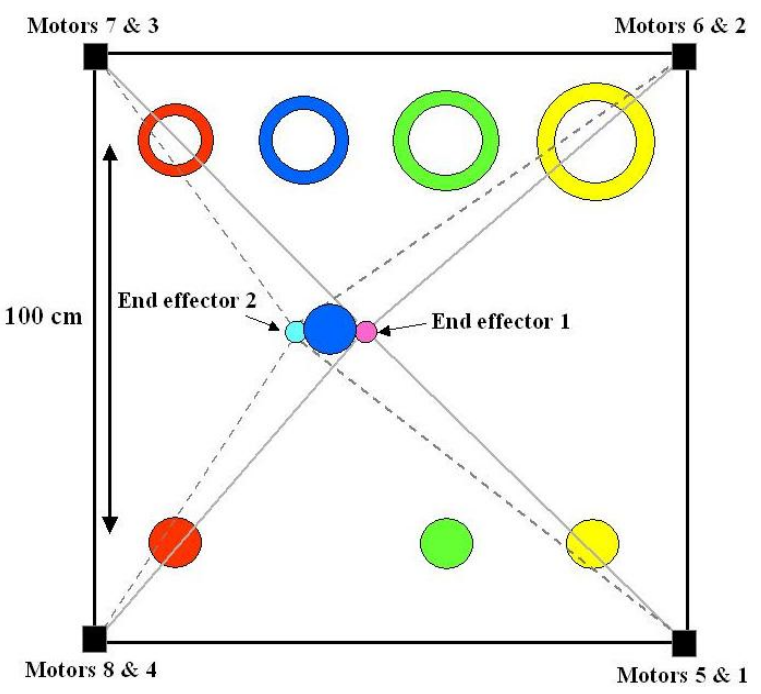

Figure 1: Illustration of the virtual environment.

it. CHASE allows users to locate and grab their collaborator using haptics [14]. In [16] a virtual environment that allows two users to collaboratively sculpt from remote locations, has been presented. Here haptic feedback is used to render the tool's pressure on the clay and to avoid the simultaneous editing of a vertex. Similarly Chan et al. [4] have reported the use of vibro-tactile cues to facilitate turn-taking in an environment that support collaboration but only one user remains in control and has the rights to manipulate objets at a particular instant. Virtual Fixtures (VFs) formalism has been presented in [26] and mechanics based characterization and design guidelines have been reported in [19]. Collaborative work that requires tight coupling between the users has not been studied in the context of haptic guides.

\section{Description OF the SyStem}

In this section we present a system that enables two users, to cooperatively manipulate virtual objects using string-based simulated parallel robots in a VE. Secondly we present models of the proposed haptic guides that may assist the cooperative manipulation of objects.

The VE for cooperative manipulation has a simple cubic structure (each side is $120 \mathrm{~cm}$ ), consisting of three walls, floor and ceiling. Furthermore the VE contains four cylinders each with a distinct color and standing lengthwise in a line (see figure 1). In front of each cylinder at a distance of $100 \mathrm{~cm}$ there is a torus of the same color. All cylinders have the same radii of $4.5 \mathrm{~cm}$. The red, green, blue and yellow toruses have inner radii $4.6 \mathrm{~cm}, 4.8 \mathrm{~cm}, 5.0 \mathrm{~cm}$ and $5.20 \mathrm{~cm}$ respectively. Cylinders and toruses have $12 \mathrm{~cm}$ distance between them. We have modeled two SPIDAR (3DOF) to be used as robots [20]. At each corner of the cube a motor for one of the SPIDAR has been mounted. The end effectors of the SPIDARs have been represented by two spheres of distinct colors. The movements of these spheres are controlled by the real SPIDARs. Each end effector uses 4 wires (represented by dashed and smooth lines) for connection with its corresponding motors. Therefore, users' movements are constrained by the wire arrangement of the SPIDAR.

One of the important tasks related to collaborative/cooperative system is the representation of users in the virtual world. This is normally carried out using avatars $[15,15,10,7]$ or some other representations like virtual hands or balls $[5,6,12]$. We use two spheres which are identical in size but different in colors (one is violet and the other is blue) in order to have users'co-presence in VE. In order to lift and/or transport a cylinder the violet end effector

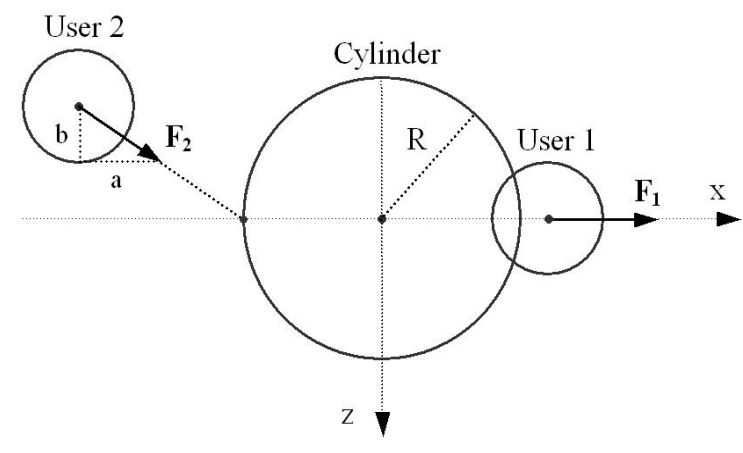

Figure 2: Illustration of the attractive haptic guide: view from top.

will always rest on right and blue on left of the cylinder.

\subsection{Models of the Haptic Guides}

Cooperative work is really a challenging research area, because there are many points to be treated. For example the sense of copresence and awareness may have profound effects on cooperation. Similarly the cooperating persons should also have some feedback to know when they can start together, can leave each other (when task is finished), or if there is some interruption during task. In this context we propose dynamic haptic guides.

\subsubsection{Attractive Haptic Guide}

If any user moves to touch a cylinder on its proper side, and if the second user's end effector's height corresponds to a point on y-axis of the cylinder then the second user will feel a force of attraction toward the cylinder (see figure 2. The attractive guide serves two purposes, firstly it lets know a user that his collaborator is in contact with the cylinder. Secondly it helps to keep intact the two pointers with cylinder during transportation if one of the users looses control of the cylinder then he is immediately brought back by the attractive force.

Here the positions of cylinder, user 1 and user2 are represented by equation 1, 2 and 3 respectively.

$$
\begin{aligned}
\operatorname{POS}_{c y l} & =\left(X_{c y l}, Y_{c y l}, Z_{c y l}\right) \\
\operatorname{POS}_{u 1} & =\left(X_{u 1}, Y_{u 1}, Z_{u 1}\right) \\
\operatorname{POS}_{u 2} & =\left(X_{u 2}, Y_{u 2}, Z_{u 2}\right)
\end{aligned}
$$

Similarly "R" and "r" represent the radii of the cylinders and spheres (3D cursors) respectively. $\mathrm{K}$ is a constant. The attractive force is calculated as:

$$
\begin{gathered}
\vec{F}_{1}=K\left[\left(X_{u 1}-r\right)-\left(X_{c y l}+R\right)\right] \vec{u}_{x} \\
\left|\vec{F}_{2}\right|=\left|\vec{F}_{1}\right| \\
\vec{F}_{2}=a \vec{u}_{x}+b \vec{u}_{z}
\end{gathered}
$$

Where

$$
a=\frac{\left|\vec{F}_{2}\right| \cdot\left[X_{u 2}-\left(X_{c y l}-R\right)\right]}{\sqrt{\left(Z_{c y l}-Z_{u 2}\right)^{2}+\left[\left(X_{c y l}-R\right)-X_{u 2}\right]^{2}}}
$$

and 


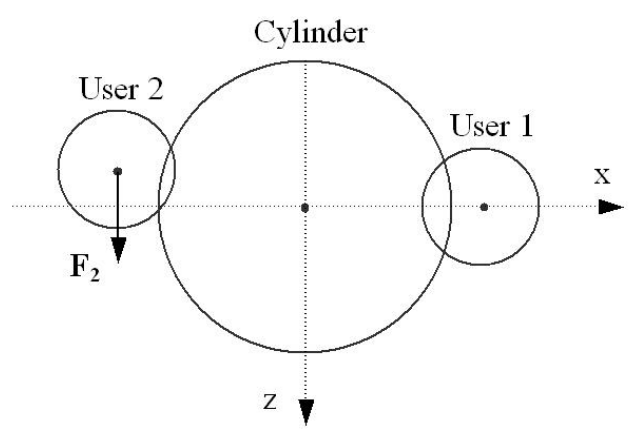

Figure 3: Illustration of the speed control haptic guide :view from top.

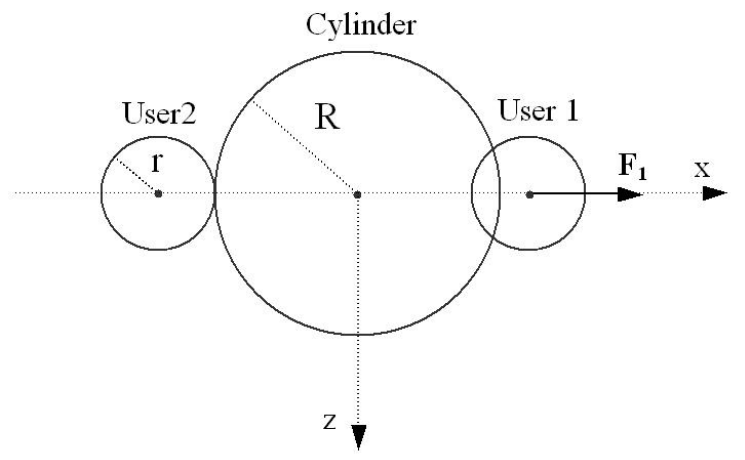

Figure 4: Illustration of the penetration control haptic guide : view from top.

$$
b=\frac{\left|\vec{F}_{2}\right| \cdot\left(Z_{c y l}-Z_{u 2}\right)}{\sqrt{\left(Z_{c y l}-Z_{u 2}\right)^{2}+\left[\left(X_{c y l}-R\right)-X_{u 2}\right]^{2}}}
$$

\subsubsection{Speed control Haptic Guide}

During the previous experiments over cooperative manipulation of objects we have observed that users usually loose control of the object when there is considerable difference between the speed of the two users while transporting it. In order to smoothly transport the object and minimize errors we propose another guide called "Speed control haptic guide" that slightly blocks the user whose speed exceeds a threshold as compared to his collaborator (see figure 3). The speed control force is calculated according to equation 9.

$$
\vec{F}_{2}=K\left(Z_{u 1}-Z_{u 2}\right) \vec{u}_{z}
$$

Here $\vec{F}_{2}$ is blocking force felt by user 2 because he/she is moving to fast as compared to his/her collaborator (see figure 3 ).

\subsubsection{Simple force feedback}

The penetration control haptic guide allows the users to feel a blocking force as a function of their end effector penetration into the cylinder. This force not only increases realism of the task but also helps users to smoothly transport the object. The penetration control force is calculated according to equation 10 . Here $\vec{F}_{2}$ is zero because the end effector of user 2 has no penetration (see figure 4).

$$
\vec{F}_{1}=K\left[\left(X_{u 1}-r\right)-\left(X_{c y l}+R\right)\right] \vec{u}_{x}
$$

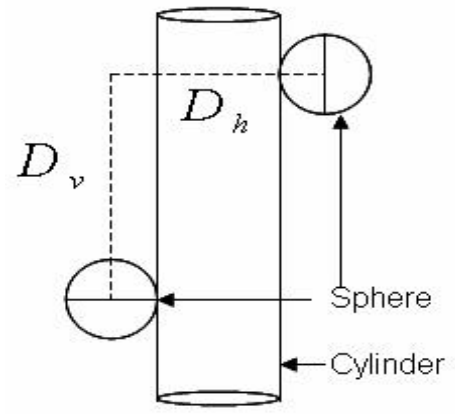

Figure 5: Illustration of constraints for cooperative manipulation.

$$
\vec{F}_{2}=0
$$

With each haptic guide the following conditions are also checked once the two end effectors touch a cylinder ( Fig. 5).

$$
\begin{gathered}
D_{h} \geq 2 R_{c}-K \\
D_{v} \leq T
\end{gathered}
$$

In equation $12, D_{h}$ represents the horizontal distance between the centers of the two spheres, $R_{c}$ is the radius of the cylinder and $K$ is a positive constant. This check ensures that the spheres must not completely penetrate the cylinder and should remain visible during the task. In equation 13, $D_{v}$ represents the vertical distance between the centers of the two spheres that must be less than or equal to a threshold $T$. When conditions in equation 12 and equation 13 are both satisfied then users can cooperatively move the cylinder.

\subsection{Experimental Setup}

This section presents the VR platform called VIREPSE (Virtual Reality Platform for Simulation and Experimentations) used for experimentations. We have installed the software on pentium 4 type personal computer. The machine has $2 \mathrm{GHZ}$ processor $(5130 \mathrm{BI}-$ $\mathrm{XEON}$ ) and 4GB memory. The system is equipped with powerful graphic card (NVIDIA). We use a large $(2 \mathrm{~m} \times 2.5 \mathrm{~m})$ rear-projected screen for display and polarized glasses for stereoscopic viewing.

Similarly each user uses a real human scale SPIDAR (3DOF) to control the movement of the end effector of the virtual SPIDAR (simulated robot) (see figure 6). The SPIDAR system uses a SH4 controller from the Cyverse Inc. In order to provide force feedback to both users, a total of 8 motors are placed on the corners of a cubic frame surrounding the users. The system uses RE10 DC motors from Maxon Inc., pulleys and optical encoders. Each end of the hand attachment is wrapped around a pulley driven by a DC motor. Using the tension and length of each string, the system generates appropriate forces. The controller of SPIDAR uses USB for connection with computer. We developed the software using $\mathrm{C}++$ and OpenGL Library.

\section{EXPERIMENTATION}

\subsection{Procedure}

In order to evaluate the system and investigate the effect of haptic guides on user performance in cooperative object manipulation, we carried out user experimentation. For this purpose a group of ten male volunteers participated. They were Master and $\mathrm{PhD}$ students and aged from 22 to 35 . All the participants performed the experiment with the same person who was expert of the domain and also of the proposed system. 


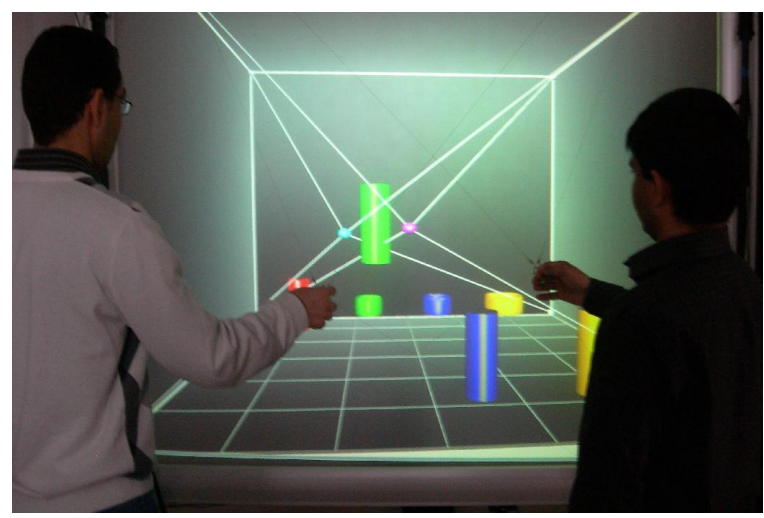

Figure 6: Illustration of the cooperative task in LISA VR platform.

Each subject was given a short briefing about the experiment. They were also given a pre-trial in which they experienced all feedback to get them familiar with the system. On launching the application, users could see the two end effectors (violet and blue spheres attached to the wires) of the robots (virtual SPIDARs) on screen. The violet sphere was assigned to the expert while the subjects were in charge of the blue one. In order to pickup the cylinder the expert needs to touch it from right while the subject should rest on its left. The experiment was carried out under the following four conditions.

- $\mathrm{C} 1=$ No force feedback

- $\mathrm{C} 2=$ Simple force feedback

- $\mathrm{C} 3=$ Attractive haptic guide

- $\mathrm{C} 4=$ Speed control haptic guide

All the ten subjects performed the experiment using distinct counter balanced combinations of the four conditions. We recorded the task completion time for each cylinder. The time counter started for a cylinder once the two end effectors had an initial contact with it, and stopped when it is properly placed in the torus. The indicator for the proper placement of cylinder was change in color (white) of the torus. Similarly we recorded the number of times the cylinder was dropped as errors. After task completion we gave each user a questionnaire in order to have the subjective feedback.

\subsection{Task}

The experiment for the users was to pick up a cylinder cooperatively and put it into the torus whose color matches with the cylinder. The users were required to place all the cylinders in their corresponding toruses in a single trial. Each group performed exactly five trials under each condition. Thus each user had 80 manipulations of cylinders under all conditions. The order of selection of the cylinders was also the same for all groups i.e to start from the red, go on sequentially and finish at yellow (right).

In following subsections we not only present and analyze the results of task completion time but also the error made during task accomplishment. Similarly the user's responses collected through questionnaire is also thoroughly examined and discussed.

\subsection{Task completion time}

For task completion time the ANOVA $(\mathrm{F}(3,9)=10.01, \mathrm{p}<0.05)$ is significant. Comparing the task completion time of $\mathrm{C} 1$ and $\mathrm{C} 2$, we

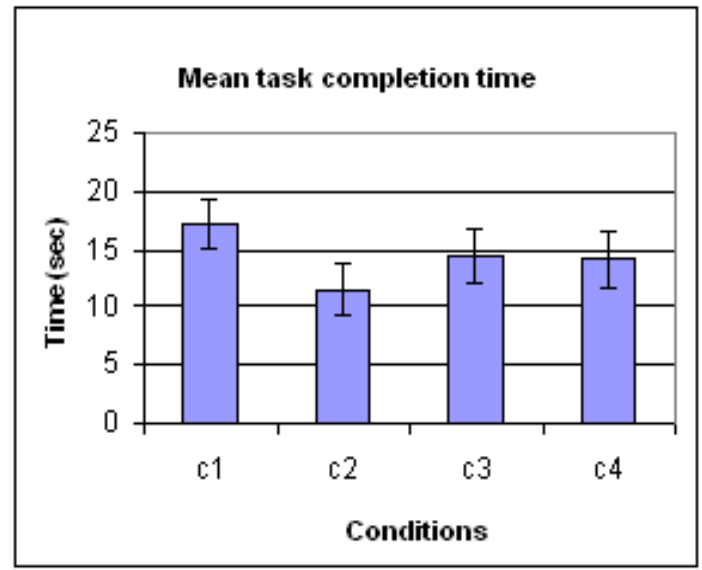

Figure 7: Task completion time under experimental conditions.

have $17.24 \mathrm{sec}(\mathrm{std} 2.09)$ and $11.61 \mathrm{sec}$ (std 2.35) respectively with a significant ANOVA. Comparing C1 (17.24 sec, std 2.09) with C3 (14.43 sec, std 2.31) also gives significant result. Similarly the comparison of C1 (17.24 sec, std 2.09) with C4 (14.27 sec, std 2.41) also gives significant ANOVA. These results show that haptic guides have an influence and increase users' performance in cooperative manipulation of objects.

Now we compare C2 (17.24 sec std 2.09) with C3 (14.43 sec std 2.31), the ANOVA result is significant. Similarly comparing C2 (17.24 sec std 2.09) with C4 (14.27 sec std 2.41) also gives significant ANOVA. On the other hand the comparison of C3 (14.43 sec std 2.31) with C4 (14.27 sec std 2.41) does not give a significant result. These results show that users performed better in condition $\mathrm{C} 2$ as compare to conditions $\mathrm{C} 3$ and $\mathrm{C} 4$. We got the same level of performance under conditions $\mathrm{C} 3$ and $\mathrm{C} 4$ (see figure 7). These results show that haptic guides enabled the users to achieve better task cooperation and decrease errors.

\subsection{Error in task completion}

When one or both users were detached from the cylinder during task accomplishment, it was considered as an error. We recorded the number of errors for each cylinder under each condition. we present a global error analysis for each condition (see figure 8). Here $\mathrm{C} 1$ has average of 2.1 errors with std 0.86 . Similarly C2, $\mathrm{C} 3$ and $\mathrm{C} 4$ have errors of 0.76 (std 0.62), 1.74 (std 0.55) and 1.32 (std 0.68) respectively. C2 and C4 have significantly low errors as compared to $\mathrm{C} 1$. Similarly $\mathrm{C} 2$ has also significantly low errors as compared to $\mathrm{C} 3$.

\subsection{Subjective evaluation}

In this section we analyze the responses collected through questionnaire. The questionnaire had four questions with three to four options for response. For each question the subjects had to select an option.

- Q1: What condition did you prefer?
(a) $\mathrm{C} 1$ (b) $\mathrm{C} 2$ (c) $\mathrm{C} 3$ (d) $\mathrm{C} 4$

For that question $40 \%$ subjects preferred C2 while $30 \%$ opted for $\mathrm{C} 3$ and $30 \%$ for $\mathrm{C} 4$.

- Q2: Which feedback did you find the most pertinent?

(a) $\mathrm{C} 1$ (b) $\mathrm{C} 2$ (c) $\mathrm{C} 3$ (d) $\mathrm{C} 4$

To that question, conditions $\mathrm{C} 1, \mathrm{C} 2, \mathrm{C} 3$ and $\mathrm{C} 4$ obtained the preference of $0 \%, 30 \%, 30 \%$ and $40 \%$ subjects respectively. 


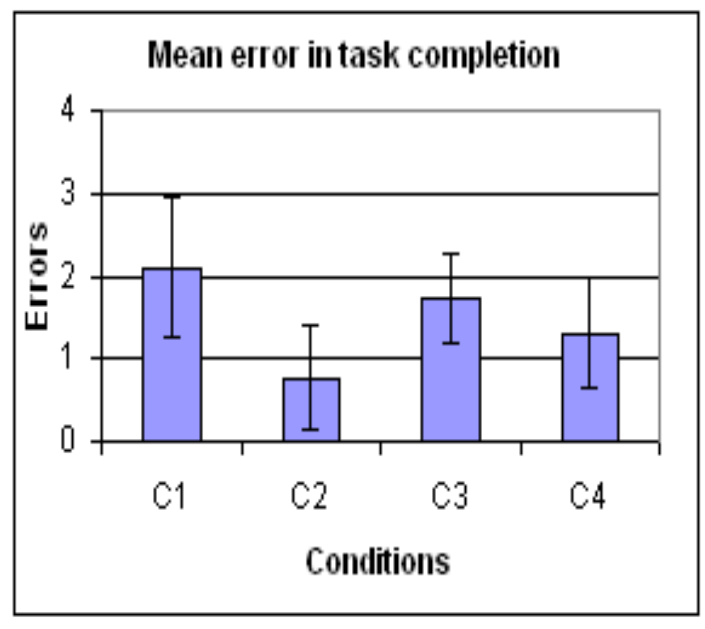

Figure 8: Illustration of error for various conditions.

- Q3: Which part of the task did you find the most difficult?

(a) Object picking (b) Transportation (c) Placement of object.

According to $70 \%$ of the subjects the most difficult part of the task was transportation. The remaining 30\% marked the placement of object as the most difficult part of the task.

- Q4: In which condition did you sense more the presence of your collaborator?

\section{(a) $\mathrm{C} 1$ (b) $\mathrm{C} 2$ (c) $\mathrm{C} 3$ (d) $\mathrm{C} 4$}

To that question $40 \%$ of the subjects better sensed the presence of their collaborator in condition $\mathrm{C} 4$. The opinion of $60 \%$ was equally divided for conditions $\mathrm{C} 2$ and $\mathrm{C} 3$. According to users' responses to the questionnaire and remarks we observed that all haptic guides significantly enhanced users' cooperation and awareness during task execution and thus resulted in better performance.

\subsection{User learning}

Learning is defined here by the improvement of group performance during task repetitions. We asked each group to repeat 5 times the previously defined task. The results show that applying condition $\mathrm{C} 1$, the subjects completed the task in $19.4 \mathrm{sec}$ during the first trial and in $15.3 \mathrm{sec}$ during the fifth trial. They completed the task under condition $\mathrm{C} 2$ in a mean time of $13.62 \mathrm{sec}$ in the first trial, while it took $10 \mathrm{sec}$ in the fifth trial. In condition $\mathrm{C} 3$, they completed the task in 16 sec during the first trial and in 13.04 sec during the fifth trial. Similarly we have mean time of $17.05 \mathrm{sec}$ under condition C4 for the first trial and $12 \mathrm{sec}$ for the last trial. (see figure 9).

This results in performance improvement of $21.13 \%, 26.58 \%$, $18.5 \%$ and $29.62 \%$ for conditions $\mathrm{C} 1, \mathrm{C} 2, \mathrm{C} 3$ and $\mathrm{C} 4$ respectively.

\section{CONCLUSION AND FUTURE WORK}

In this paper we simulate the use of two string based parallel robots in cooperative teleoperation task. Two users standing in front of a large screen operate each robot. We propose three haptic guides' models, and investigate their effects on cooperation, co-presence, awareness and users performance. In addition we also examined the effect of simple force feedback in cooperative work. Ten volunteers cooperatively performed a peg-in-hole task. Results have revealed that haptic guides have a significant effect on task execution. They not only increase users performance but also enhance the sense of co-presence and awareness.

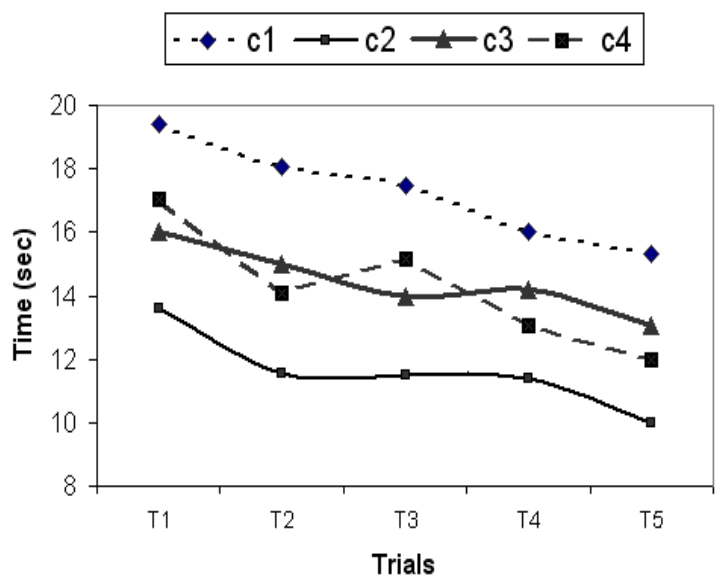

Figure 9: Illustration of user learning in various conditions.

Moreover we got best user performance with simple force feedback while attractive and speed control haptic guides gave the same level of performance. Our investigations will help in the development of teleoperation systems for cooperative assembly, maintenance, surgical training and rehabilitation systems.

In order to examine the effect of our proposed guides in cooperative work, we will carry out two experiments in the network environment where the two users will not co-locate. In the first experiment we will use a phantom on each side while in the second experiment there will be two SPIDARs.

\section{REFERENCES}

[1] J. Allard, S. Cotin, F. Faure, P.-J. Bensoussan, F. Poyer, C. Duriez, H. Delingette, and L. Grisoni. Sofa :an open source framework for medical simulation. In Medicine Meets Virtual Reality (MMVR), 2007.

[2] C. Basdogan, C. hao Ho, M. A. Srinivasan, and M. Slater. An experimental study on the role of touch in shared virtual environments. In ACM Transactions on Computer-Human Interaction, 2000.

[3] C. Basdogan, C.-H. Ho, M. A. Srinivasan, and M. Slater. Virtual training for a manual assembly task. In Haptics-e, volume 2, 2001.

[4] A. Chan, K. Maclean, and J. Mcgrenere. Designing haptic icons to support collaborative turn-taking. International Journal of HumanComputer Studies, 2007.

[5] J. W. Chastine, J. C. Brooks, Y. Zhu, G. S. Owen, R. W. Harrison, and I. T. Weber. Ammp-vis: a collaborative virtual environment for molecular modeling. In VRST '05: Proceedings of the ACM symposium on Virtual reality software and technology, pages 8-15, New York, NY, USA, 2005. ACM.

[6] M. David, B. Arnaldi, and N. Plouzeau. A general framework for cooperative manipulation in virtual environments. Virtual Environments'99 Proceedings of the Eurographics Workshop, pages 169-178, 1999.

[7] C. Eckes, K. Biatov, F. Hlsken, J. Khler, P. Breuer, P. Branco, and L. Encarnacao. Towards sociable virtual humans: Multimodal recognition of human input and behavior. The International Journal of Virtual Reality, 6(4):21-30, 2007.

[8] D. M. Gaba. The future vision of simulation in health care. In Qual Saf Health Care, 2004.

[9] C. Greenhalgh and S. Benford. Massive: a collaborative virtual environment for teleconferencing. ACM Transactions on Computer Human Interaction, 2(3):239-261, 1995.

[10] I. Heldal, M. Spante, and M. Connell. Are two heads better than one?: object-focused work in physical and in virtual environments. VRST '06: Proceedings of the ACM symposium on Virtual reality software and technology, pages 287-296, 2006. 
[11] J. Jordan, J. Mortensen, M. Oliveira, M. Slater, B. K. Tay, J. Kim, and M. A. Srinivasan. Collaboration in a mediated haptic environment. The 5th Annual International Workshop on Presence, 2002.

[12] J. Mortensen, V. Vinayagamoorthy, M. Slater, A. Steed, B. Lok, and M. C. Whitton. Collaboration in tele-immersive environments. In Proceedings of the workshop on Virtual environments, pages 93-101, 2002.

[13] U. B. I. S. M. B. F. S. N. Magnenat-Thalmann, P. Volino and F.-E. Wolter. From physics-based simulation to the touching of textiles: The haptex project. The International Journal of Virtual Reality, 6(3):3544, 2007.

[14] I. Oakley, S. Brewster, and P. Gray. Can you feel the force? an investigation of haptic collaboration in shared editors. In In Proceedings of Eurohaptics, pages 54-59, 2001.

[15] O. Otto, D. Roberts, and R. Wolff. A review on effective closelycoupled collaboration using immersive cve's. In VRCIA '06: Proceedings of the 2006 ACM international conference on Virtual reality continuum and its applications, pages 145-154. ACM, 2006.

[16] B. Plimmer, A. Crossan, S. A. Brewster, and R. Blagojevic. Collaborative virtual sculpting with haptic feedback. In Virtual Reality, volume 10, pages 73-83. Springer London, 2006.

[17] B. Plimmer, A. Crossan, S. A. Brewster, and R. Blagojevic. Multimodal collaborative handwriting training for visually-impaired people. In CHI '08: Proceeding of the twenty-sixth annual SIGCHI conference on Human factors in computing systems, pages 393-402, New York, NY, USA, 2008. ACM.

[18] G. V. Popescu, G. Burdea, and R. Boian. An experimental study on the role of touch in shared virtual environments. In ACM Transactions on Computer-Human Interaction, volume 7, 2000.

[19] R. Prada and S. Payandeh. On study of design and implementation of virtual fixtures. Virtual Reality, 13(2):117-129, 2009.

[20] P. Richard, D. Chamaret, F.-X. Inglese, P. Lucidarme, and J.-L. Ferrier. Human-scale virtual environment for product design: Effect of sensory substitution. International Journal of Virtual Reality, 5(2):3744, 2006.

[21] G. Riva, M. Bacchetta, G. Cesa, and E. Molinari. Cybertherapy: Internet and virtual reality as assessment and rehabilitation tools for clinical psychology and neuroscience. In IOS Press, pages 121-164, 2004.

[22] B. W. S. Marks, J. Windsor. Collaborative soft object manipulation for game engine-based virtual reality surgery simulators. Proceedings of Image and Vision Computing, page 205210, 2007.

[23] E.-L. Sallnas, K. Rassmus-Grohn, and C. Sjostrom. Supporting presence in collaborative environments by haptic force feedback. $A C M$ Trans. Comput.-Hum. Interact., 7(4):461-476, 2000.

[24] X. Shen, F. Bogsanyi, L. Ni, and N. Georganas. A heterogeneous scalable architecture for collaborative haptics environments. Proceedings of the 2nd IEEE Internatioal Workshop on Haptic, Audio and Visual Environments and Their Applications, pages 113 - 1187, 2003.

[25] S. Shirmohammadi and N. D. Georganas. An end-to-end communication architecture for collaborative virtual environments. Comput. Netw., 35(2-3):351-367, 2001.

[26] S.Otmane, M.Mallem, A.Kheddar, and F.Chavand. Active virtual guide as an apparatus for augmented reality based telemanipulation system on the internet. IEEE Computer Society, pages 185-191, 2000.

[27] S. Ullah, N. Ouramdane, S. Otmane, P. Richard, F. Davesne, and M. Mallem. Augmenting $3 \mathrm{~d}$ interactions with haptic guide in a large scale virtual environment. In VRCAI '08: Proceedings of The 7th ACM SIGGRAPH International Conference on Virtual-Reality Continuum and Its Applications in Industry, pages 1-6, New York, NY, USA, 2008. ACM. 